\title{
Does ultrasound measurement improve the accuracy of electronic brachytherapy in the treatment of superficial non-melanomatous skin cancer?
}

\author{
Uma Goyal, MD!, Junhan Pan, MS, PSM!', Haiyan Cui, PhD², Baldassarre Stea, MD, PhDl \\ 'Department of Radiation Oncology, University of Arizona, Tucson, AZ, ${ }^{2}$ Division of Biostatistics, University of Arizona Cancer Center, Tucson, AZ, USA
}

\begin{abstract}
Purpose: Electronic brachytherapy (eBT) is a form of contact radiation therapy used for thin superficial non-melanomatous skin cancers (NMSCs). An accurate measurement of diameter and depth is important for eBT treatment planning. Therefore, we compared clinical measurements by an experienced physician to measurements obtained using ultrasound (US), an objective imaging modality, in order to determine if clinical measurements were accurate enough for adequate NMSC treatment.

Material and methods: Eighteen patients with 20 biopsy-proven NMSCs first had a clinical examination and then an US evaluation prior to starting eBT. One physician provided a clinical measurement for diameter and depth based on physical examination during radiation oncology consultation. The patients then had an US evaluation with a 14 or $18 \mathrm{MHz}$ US unit, to determine both the diameter and depth measurements; eBT dose prescription was done using the US derived measurements. The clinical measurements and US measurements were compared using a $t$-test.

Results: Seventeen lesions were basal cell carcinoma and 3 lesions were squamous cell carcinoma. The most common location was the nose (10 lesions). The difference between the clinical and the US derived measurements for the second largest diameter was found to be statistically significant $(p=0.03)$, while the difference for the largest diameter of the lesions was not $(p=0.24)$. More importantly, the depth measurements obtained with US were also found to be significantly different from the clinical estimates $(p=0.02)$. All patients have had a complete response to therapy with a median follow-up of 24 months.

Conclusions: Statistically different measurements were obtained in 2 of 3 parameters used in choosing applicator size and prescription depth using an US assessment. The data presented suggests that US is a more objective modality than clinical judgment for determining superficial NMSC diameter and prescription depth for personalized eBT planning.

J Contemp Brachytherapy 2017; 9, 1: 14-19 DOI: https://doi.org/10.5114/jcb.2017.65476
\end{abstract}

Key words: electronic brachytherapy, skin cancer, ultrasound.

\section{Purpose}

There are multiple treatment options for superficial non-melanomatous skin cancers (NMSC) such as surgery, topical chemotherapies, and radiotherapy $[1,2,3,4,5,6]$. The method of treatment assumes an even greater importance when these lesions are located in cosmetically sensitive parts of the body, such as the face, nose, ears, and eyelids.

There are several different radiotherapy techniques that can be used to effectively treat NMSCs. When treatment with conventional megavoltage techniques (electrons or photons) is chosen, a much larger surface area is commonly treated in order to account for electronic equilibrium, patient setup, and penumbra effect. Treatments are usually protracted over many weeks $[5,7,8]$, and in order to ensure adequate dose to the skin, a water-based custom bolus is often utilized. Clinical estimates of lesion depth or a default depth for all lesions, especially for shallow tumors, are commonly used $[5,9,10]$. In such cases, the precise depth of the lesion is not critical because the high-energy beam more than compensates for the uncertainty of depth. Other radiation treatment modalities used for skin cancer include: highdose-rate (HDR) brachytherapy involving molds, flaps, or radionuclide applicators $[2,4,11,12,13,14,15]$, orthovoltage radiation $(40-300 \mathrm{kVp})[16,17,18]$, both of which have been successfully used to treat skin cancers in 6-30 fractions. Of course, when skin cancer is highly invasive such as in the case of perineural invasion, more sophisticated approaches (CyberKnife) have been used [19].

Electronic brachytherapy (eBT) has also been shown to be effective for very superficial lesions $[20,21,22,23,24]$. The Xoft eBT skin applicators commonly used in practice are available in 4 different sizes $(10,20,35$, and $50 \mathrm{~mm})$.
Address for correspondence: Uma Goyal, MD, Department of Radiation Oncology, University of Arizona, 1501 N. Campbell Ave. Tucson, AZ 85724, USA, phone: +1 520.626.0434, fax: +1 520.626.2032, e-mail: ugoyal@email.arizona.edu
Received: 26.10 .2016

Accepted: 19.12 .2016

Published: 28.02.2017 
These applicators are placed directly in contact with the lesion, thus the name of contact radiotherapy $[25,26]$. This technique does not require a radioactive isotope, but uses a similar hypofractionated regimen as other orthovoltage or brachytherapy techniques previously mentioned $[7,20,25,26]$. Excellent local control of $98.7 \%$ and cosmesis of $94.2 \%$ at a minimum follow-up of 16.1 months have been reported for NMSC treated with eBT [10].

Accurate prescription depth and cross sectional measurements of the tumor are necessary for all the various treatment modalities described above, not only to adequately treat the target, but also to prevent unnecessary skin toxicity [27]. Particularly for brachytherapy-based approaches, flaps and molds are used, which have catheters placed $1 \mathrm{~cm}$ apart and about $5 \mathrm{~mm}$ from the skin surface, so that dwell positions of the radioactive source do not cause overdose to the skin surface, due to high surface doses. When using eBT, however, the maximum prescription depth is usually kept at about $<5 \mathrm{~mm}$ as at that level the surface dose is approximately $200 \%$ of the depth dose as described in previously published data [20]. Thus, surface applicators such as for the radionuclide technique and eBT can lead to a higher surface dose with an increasing depth [20]. Such high surface dose can lead to skin radionecrosis and/or prolonged healing time. Therefore, for tumors approaching or thicker than $4 \mathrm{~mm}$, perhaps another modality such as conventional photon or electron radiotherapy should be used, especially when they occur in the lower extremities where healing is not optimal due to poor blood perfusion. Previous investigators have used their clinical judgment in determining the diameter and depth of NMSC lesions $[5,9,10]$, but this method can be quite subjective and imprecise.

To our knowledge, there are no publications comparing clinical measurements to US measurements for NMSC treated with eBT. In this study, an objective method to measure pre-treatment NMSCs using US imaging was hypothesized to provide significantly better 3-dimension (3D) measurements, compared to clinical estimates of tumor measurements from physical examination or pathology reports. Here, we report the results of our comparative study.

\section{Material and methods}

From December 2013 to April 2015, 19 patients harboring 22 biopsies confirmed NMSCs lesions were treated with definitive eBT (Axxent eBx; Xoft - a subsidiary of iCAD, Inc., Sunnyvale, CA, USA). Eighteen patients with 20 lesions were eligible for this study having both clinical and US measurements available. These skin lesions included biopsy proven basal cell carcinoma (BCC) or squamous cell carcinoma (SCC). Due to limitation of the eBT technique (low energy of $50 \mathrm{kVp}$ ), the depth of lesions treated was limited to $<5 \mathrm{~mm}$. In addition, the diameter of the largest applicator available is $50 \mathrm{~mm}$, so the largest diameter dimension of the lesion was limited to $35 \mathrm{~mm}$, if a 7-mm radial margin is included to account for microscopic disease (CTV) and setup uncertainty (PTV). One experienced physician obtained the clinical measurements during physical examination prior to obtaining the
US. Then the patient was referred to medical imaging to get an US derived measurement. Radiation oncology and radiology records, referring physician documentation, and hospital records were examined within an accessible electronic medical record. This retrospective study was approved by our institutional review board.

\section{Patient characteristics}

Eighteen patients with 20 NMSCs had both a clinical assessment for measurements of lesions by the treating radiation oncologist and an US evaluation with measurements of the lesions prior to commencing eBT. The mean age of these patients was 70 years old. Fifty percent of patients were female. The majority of patients $(85 \%)$ had BCC. The most common location was the nose (10 lesions), followed by the cheek (3), forehead (2), ear (2), scalp (1), eyebrow (1), and extremities (1). Table 1 summarizes both patient and lesion characteristics.

Table 1. A summary of both patient and lesion characteristics. The maximum value for each measurement is shown for the largest diameter, second largest diameter, and depth

Factor Data

\begin{tabular}{|c|c|}
\hline Age mean, years (range) & $70(54-85)$ \\
\hline Females, $n(\%)$ & $9(50)$ \\
\hline Males, $n(\%)$ & $9(50)$ \\
\hline \multicolumn{2}{|l|}{ Lesion characteristics, $n$ (\%) } \\
\hline Basal cell carcinoma & $17(85)$ \\
\hline Squamous cell carcinoma & $3(15)$ \\
\hline Nose & $10(50)$ \\
\hline Cheek & $3(15)$ \\
\hline Forehead & $2(10)$ \\
\hline Ear & $2(10)$ \\
\hline Scalp & $1(5)$ \\
\hline Eyebrow & $1(5)$ \\
\hline Extremities & $1(5)$ \\
\hline \multicolumn{2}{|c|}{ Maximum values for each measurement (mm) } \\
\hline \multicolumn{2}{|l|}{ Largest diameter } \\
\hline Clinical & 20 \\
\hline Ultrasound & 17 \\
\hline \multicolumn{2}{|l|}{ Second largest diameter } \\
\hline Clinical & 15 \\
\hline Ultrasound & 11 \\
\hline \multicolumn{2}{|l|}{ Depth } \\
\hline Clinical & 3 \\
\hline Ultrasound & 4 \\
\hline
\end{tabular}




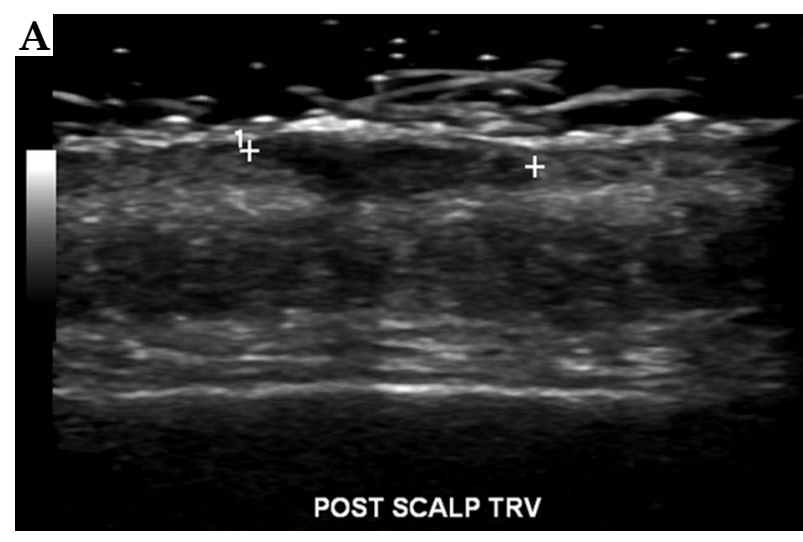

Fig. 1. Ultrasound images of a scalp basal cell carcinoma

\section{Diagnosis and measurements}

A suspicious skin lesion was first biopsied by a dermatologist. Patients were then referred to radiation oncology for consultation. Physical examination included palpation of the skin lesion for a 3D clinical assessment by the treating radiation oncologist. Patients were then sent for US measurements to obtain the maximal cross sectional diameters and the depth to be used for treatment planning.

Routine US scan was done with a LOGIQ E9 (GE Healthcare, Milwaukee, WI, USA) or S-3000 US units (Acuson; Siemens Medical Solutions, Mountain View, CA, USA) using linear array transducers with upper frequencies of 14 or $18 \mathrm{MHz}$. US was done with a $15 \mathrm{MHz}$ linear probe or $8-18 \mathrm{MHz}$ hockey stick probe to acquire images in transverse and sagittal planes. The US images were reviewed by an experienced radiologist for 3D measurements of the NMSC lesion. As described previously, for lesions that were not detectable by US, we used a prescription depth of $1 \mathrm{~mm}$ depth for eBT [20]. Figure 1 shows an example of US images of a scalp BCC.

\section{Electronic brachytherapy physics}

Treatment planning for eBT consists of calculating treatment time $T$ with the following formula:

$$
\mathrm{T}=\frac{D_{R x}(d)}{\dot{D}(\varnothing, 0) \times P D D(\varnothing, d) \times O F_{\text {cutout }}}
$$

where $D_{R x}(d)$ is the prescription dose per fraction at treatment depth $d, \operatorname{PDD}(\varnothing, d)$ is the percentage depth dose of the selected applicator with diameter of $(10 \mathrm{~mm}$, $20 \mathrm{~mm}, 35 \mathrm{~mm}$ or $50 \mathrm{~mm}$ ) at depth $d, \dot{D}(\varnothing, 0)$ is the X-ray source output factor at phantom surface for the same applicator, and $O F_{\text {cutout }}$ is the cutout factor and equals to unity unless a patient specific shielding is used. Percentage depth dose $\operatorname{PDD}(\varnothing, d)$ is provided by the vendor based on average value of ten X-ray sources with standard deviations of less than $5 \%$ at each depth. $\dot{D}(\varnothing, 0)$ is calibrated for the X-ray source with each applicator following American Association of Physicists in Medicine task group (AAPM TG) 61 protocol [28]: AAPM protocol for 40-300 kV X-ray beam dosimetry in radiotherapy and radiobiology. The in-air method is used with mini plane

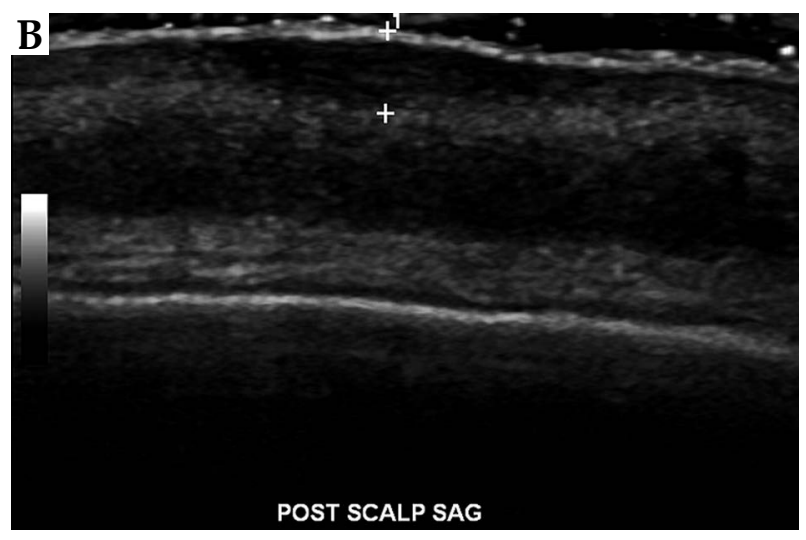

parallel plate chamber to calibrate output factor $\dot{D}(\varnothing, 0)$ for X-ray source with different applicator size (Ø) combinations at phantom surface at nominal air kerma strength $\left(A K S_{\text {nominal }}=110000\right)$. Before each treatment, treatment time $T$ will be corrected by applying correction factor $F$, which is the ratio between nominal air kerma strength $A K S_{\text {nominal }}$ and pre-treatment air kerma strength $A K S_{\text {pretx }}$. Accurate measurement of size of the tumor (lateral and vertical dimensions) is crucial to determine the applicator size $\varnothing$ and treatment depth $d$ for treatment planning.

\section{Treatment methods}

All patients were treated with a dose of 40 Gy in 10 fractions given every other day. Patients were followed up approximately 4-6 weeks after completion of eBT by their radiation oncologist with physical examination of the treated area. Patients then had routine follow-up with their dermatologist and radiation oncologist every six months for 2 years and yearly thereafter. Skin toxicity was determined using CTCAE version 4.0.

\section{Statistical analysis}

For each measurement (cross-sectional diameters and vertical depth), the number of subjects, mean, standard deviation, minimum, and maximum values were calculated. The paired t-test was used to compare the difference between clinical measurements and ultrasound measurements for the 20 NMSCs. A $p$-value $<0.05$ was considered significant. All analyses were conducted using SAS 9.4 software (Cary, NC, USA).

\section{Results}

\section{Comparison of clinical and ultrasound measurements}

On clinical examination, the 20 NMSC lesions had a mean largest diameter of $7.55 \mathrm{~mm}$, while for US the mean largest diameter was $5.79 \mathrm{~mm}(p=0.24)$. The second largest (cross-sectional) clinical dimension was also compared against US (mean $6.30 \mathrm{~mm}$ vs. $4.02 \mathrm{~mm}$, respectively), showing a statistical significance $(p=0.03)$. Furthermore, clinical measurements were found to underestimate the depth of the lesion. The mean clinical 
Table 2. The mean for the lesion dimensions is shown with statistical significance seen between the clinical and ultrasound second largest diameter and depth measurements. SE is standard error

\begin{tabular}{lccc} 
& \multicolumn{2}{c}{ Mean (SE) } & p-value for difference \\
\cline { 2 - 3 } & Clinical $(\mathrm{mm})$ & Ultrasound $(\mathrm{mm})$ & 0.24 \\
\hline Largest diameter & $7.55(5.60)$ & $5.79(4.45)$ & 0.03 \\
\hline Second largest diameter & $6.30(4.09)$ & $4.02(3.29)$ & 0.02
\end{tabular}

A

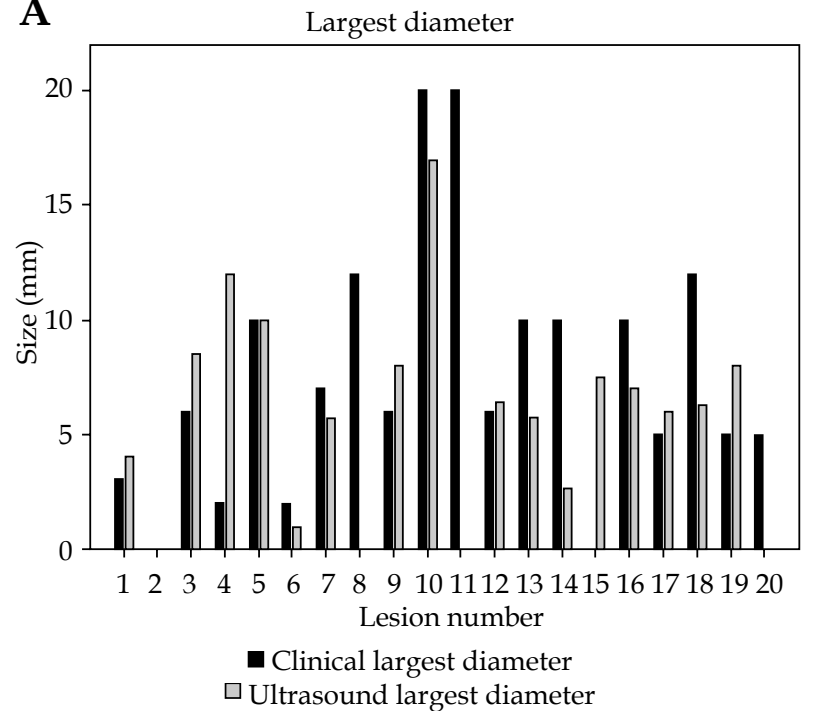

B

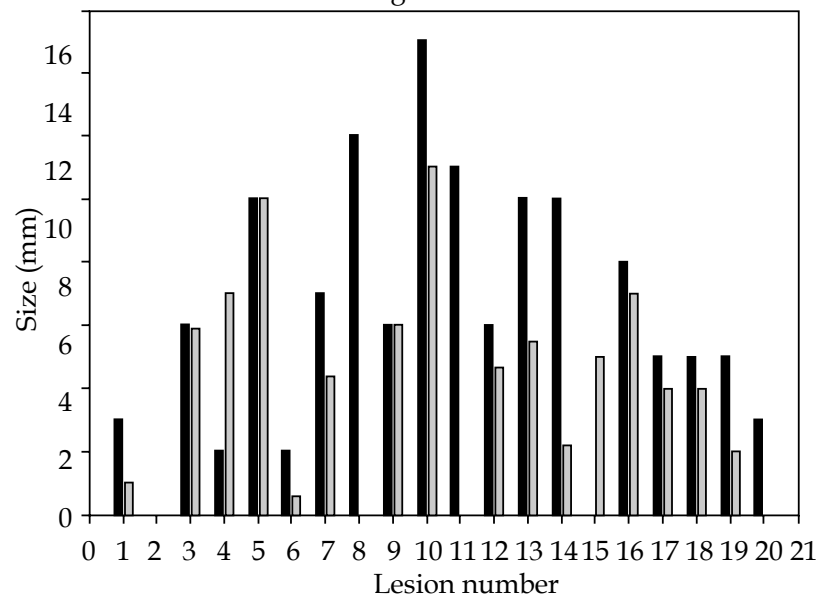

- Clinical second largest diameter

$\square$ Ultrasound second largest diameter

Fig. 2. Difference in measurements between clinical and ultrasound-derived cross sectional diameters of 20 non-melanomatous skin cancers lesions. A) Largest diameter difference $(p=0.24)$ and $(\mathbf{B})$ second largest diameter difference $(p=0.03)$

depth of the 20 NMSCs was $0.95 \mathrm{~mm}$, while the mean US derived depth was $1.76 \mathrm{~mm}(p=0.02)$. Table 1 shows the maximum value for each measurement, and Table 2 summarizes mean lesion dimensions. The difference in measurements for lesion diameter dimensions is shown in Figure 2. The difference in lesion measurements for depth is shown in Figure 3.

\section{Treatment outcomes}

With a median follow-up of 24 months, all patients have had a clinically complete response and long-term cosmetically excellent outcomes with only grade $\leq 1$ skin toxicity. No cases of skin radionecrosis or delayed healing have occurred.

\section{Discussion}

In our original feasibility paper, we described the use of US for objectively determining the diameter and depth of a pathologically proven NMSC, and the potential clinical applicability of such measurements to guide treatment planning for eBT [20]. Various clinical and imaging modalities for evaluating skin lesions, particularly melanoma, have been used for estimating the size. Dermatoscopy allows for direct magnification and visualization of a lesion providing two dimensional (2D) images from the surface but not depth $[29,30,31,32,33]$. Optical coherence tomography (OCT) uses infrared light and time-of-flight information to produce 3D images of $<1 \mathrm{~mm}$ from the skin surface $[34,35]$. Reflectance confocal microscopy (RCM) is a high cost method that uses backscatter differences for 3D images of lesions $0.5 \mathrm{~mm}$ from the skin surface to the papillary dermis [35]. However, only US imaging mo-

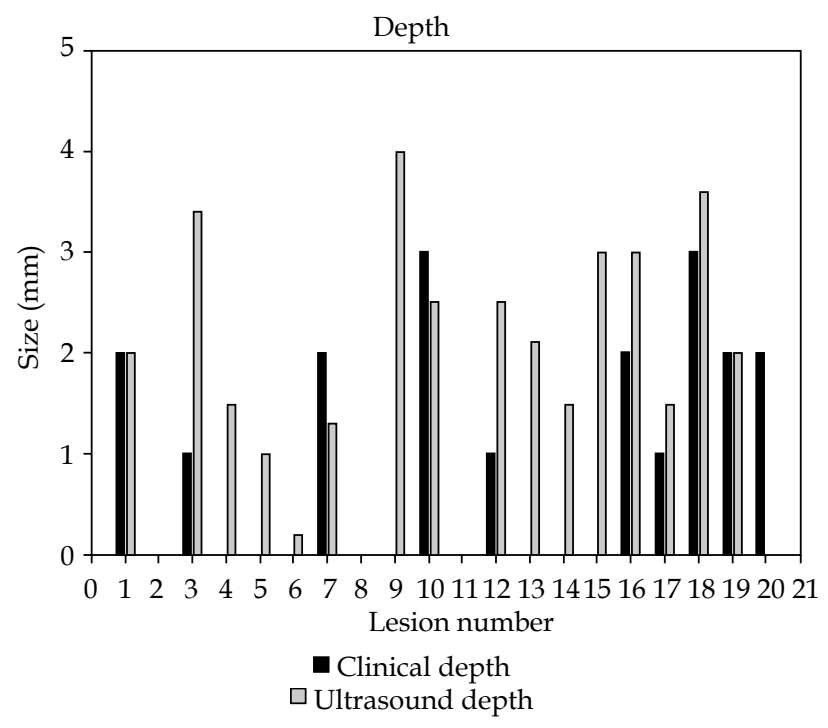

Fig. 3. Difference in depth between clinical and ultrasoundderived measurements of 20 non-melanomatous skin cancers lesions $(p=0.02)$ 
dality provides 2D and 3D images of deep dermal or subdermal layers by measuring differences in sound impedance [36], and it is commonly available in many medical centers.

Many radiation oncologists use a default depth or their clinical judgment as the sole means to determine the size of a NMSC, which can be subjective and physician-dependent $[5,9,10]$. However, US imaging provides clinicians an objective tool in estimating the size and depth of a lesion for the purpose of choosing the correct applicator size and prescription depth to be used in eBT planning and treatment. Tumors can extend subcutaneously, which may not be visually obvious or palpable. Therefore, when measurements are chosen on a purely clinical basis, the eBT applicator diameter and the prescription depth may not be adequately covering the full extent of the lesion [20].

Previously published studies evaluating the use of US for NMSCs have shown US measurement variability of $1 / 100^{\text {th }}$ of a millimeter, specifically $0.03-0.05 \mathrm{~mm}[36,37]$. Based on preoperative assessment of skin tumors and comparisons between US and histopathology for NMSCs, thickness measured by a $14-\mathrm{MHz}$ ultrasonic transducer had a good correlation for BCC $(r=0.690)$ [38]. Therefore, US compared to the gold standard of pathology showed very good correlation for the lesion's thickness [39].

Another interesting observation made in this small study is that although the means of the 20 largest diameters were not significantly different between clinical estimates and US-derived measurements, there were extreme differences in some of the individual lesion measurements (Figure 2A), which could have affected the size of the eBT applicator used. For example, if one considers lesion \#15, clinically undetectable after a shave biopsy, this lesion could have been easily treated with a $10-\mathrm{mm}$ applicator. However, on US, the largest diameter measurements of the lesion were actually $5 \times 7 \mathrm{~mm}$, thus necessitating a $20 \mathrm{~mm}$ applicator to adequately cover and treat with a margin of $7 \mathrm{~mm}$ for CTV and PTV. Obviously, the use of the wrong size applicator could translate in under-dosing the tumor, which in turn could lead to a marginal failure. Conversely, the other scenario of clinical estimates being overly generous compared to the US measurements also occurred (see case \#14 in Figure 2). Finally, in some cases, no identifiable lesion was imaged (cases \#8, 11, and 20 in Figure 2), and in these cases, the clinical measurements reflected the defect of the shave biopsy rather than real tumor. When this situation occurred, we used the clinical measurements as a default. Ultimately, because of differences noted in US derived measurements, changes in applicator size (diameter) were made in about half of the cases. This underscores the importance of relying on an objective technique such as US to estimate the correct maximal lesion diameter, rather than the clinician's tactile expertise.

Furthermore, we feel that it is very important to accurately estimate the depth of a lesion with US in order to avoid overdosing the skin surface, which can lead to soft tissue necrosis, a real risk associated with eBT, especially over the lower extremities [20]. Although the risk of complications is greater when dosing to a depth of $4-5 \mathrm{~mm}$, due to the shallow depth dose deposition of eBT, an US de- rived measurement allows the radiation oncologist to prescribe to a certain depth with confidence as necessitated by the thickness of the lesion. There is no current consensus on skin dose constraints for eBT. Cuttino et al. showed with HDR breast brachytherapy that skin dose should be within $120 \%$ of prescription dose to avoid unacceptable toxicity [40]. However, dose, fractionation, and aim for breast cancer HDR brachytherapy are not comparable to NMSC eBT. Therefore, at this moment, the clinically acceptable skin dose for NMSC eBT remains investigational. However, an accurate depth measurement eliminates "guessing" and minimizes unnecessary overdose on the surface, which can lead to ulceration, infection, poor healing, and possibly necrosis. Indeed, US measurements can be made to a tenth of a millimeter, thus allowing more precision in the depth used for eBT prescriptions.

The limitations of this study include: 1 ) the small sample size of 20 biopsies proven NMSCs, which may have led to the lack of statistical significance for the largest diameter measurements, and 2) with a median of 24 months, our follow-up is fairly short. However, no local or regional recurrences have occurred, thus far and more importantly no skin necrosis or grade 3 toxicities have been observed in these 18 patients. The significance of this contribution is that it provides radiation practitioners with a simple, low cost technique to treat very superficial lesions with adequate margins and depth of prescription rather than a best guess. No other study, to our knowledge, has described the potential variation between clinical and US measurements for NMSC eBT planning.

The need for an objective measurement of superficial NMSC diameter and depth using standard imaging such as US to eliminate guesswork at the time of prescribing and planning eBT seems to remain debatable in the radiation oncology community. However, since clinical estimates by physicians can vary, especially for depth, we feel that US measurements add to the precision and personalization of the eBT planning process.

\section{Conclusions}

The use of US is an objective modality to measure the diameter and depth of NMSC for eBT treatment planning. Depth of lesions, in particular, is important for treatment planning and can be significantly different between clinical and US measurements. With the goal of personalizing eBT treatment of very thin lesions, US can provide accurate measurements to choose the best applicator size and correct depth of prescription, which in turn prevents under- or over-dosing the tumor, and improving the chance of local control and diminishing the risk of toxicity.

\section{Disclosure}

Authors report no conflict of interest.

\section{References}

1. What you need to know about melanoma and other skin cancers. NIH Publication No 10-7625. Available at: http:/ / www. cancer.gov/cancertopics/wyntk/skin.pdf. Accessed 21 October 2014. 
2. Guix B, Finestres F, Tello JI et al. Treatment of skin carcinomas of the face by high-dose-rate brachytherapy and custom-made surface molds. Int J Radiat Oncol Biol Phys 2000; 47: 95-102.

3. Locke J, Karimpour S, Young G et al. Radiotherapy for epithelial skin cancer. Int J Radiat Oncol Biol Phys 2001; 51: 748-755.

4. Sedda A, Rossi G, Cipriani C et al. Dermatological high-doserate brachytherapy for the treatment of basal and squamous cell carcinoma. Clin Exp Dermatol 2008; 33: 745-749.

5. Lovett RD, Perez CA, Shapiro SJ et al. External radiation of epithelial skin cancer. Int J Radiat Oncol Biol Phys 1990; 19: 235-242.

6. Skowronek J. Brachytherapy in the treatment of skin cancer: an overview. Postepy Dermatol Alergol 2015; 32: 362-367.

7. Kwan W, Wilson D, Moravan V. Radiotherapy for locally advanced basal cell and squamous cell carcinomas of the skin. Int J Radiat Oncol Biol Phys 2004; 60: 406-411.

8. Rong Y, Welsh JS. Surface applicator calibration and commissioning of an electronic brachytherapy system for nonmelanoma skin cancer treatment. Med Phys 2010; 37: 5509-5517.

9. Bhatnagar A. Clinical outcomes and patient-reported outcomes following electronic brachytherapy for the treatment of non-melanoma skin cancer. Int J Radiat Oncol Biol Phys 2016; 96 (2S): E713.

10. Paravati AJ, Hawkins PG, Martin AN et al. Clinical and cosmetic outcomes in patients treated with high-dose-rate electronic brachytherapy for nonmelanoma skin cancer. Pract Rad Onc 2015; 5: e659-664.

11. Pérez-Calatayud J, Granero D, Ballester F et al. A dosimetric study of Leipzig applicators. Int J Radiat Oncol Biol Phys 2005; 62: 579-584.

12. Niu H, Hsi WC, Chu JCH et al. Dosimetric characteristics of the Leipzig surface applicators used in the high dose rate brachy radiotherapy. Med Phys 2004; 31: 3372-3377.

13. Svoboda V, Kovarik J, Morris F. High dose-rate microselectron molds in the treatment of skin tumors. Int J Radiat Oncol Biol Phys 1995; 31: 967-972.

14. Tormo A, Celada F, Rodriguez S et al. Non-melanoma skin cancer treated with HDR Valencia applicator: clinical outcomes. J Contemp Brachytherapy 2014; 6: 167-172.

15. Villalba SR, Perez-Calatayud MJ, Bautista JA et al. Novel simple templates for reproducible positioning of skin applicators in brachytherapy. J Contemp Brachytherapy 2016; 8: 344-348.

16. Marconi D, Resende B, Rauber E et al. Head and Neck Nonmelanoma Skin Cancer Treated by Orthovoltage Radiation: An Analysis of 1021 Cases. Int I Radiat Oncol Biol Phys 2014; 90: S572.

17. Kharofa J, Currey A, Wilson JF. Patient-reported outcomes in patients with nonmelanomatous skin cancers of the face treated with orthovoltage radiation therapy: a cross-sectional survey. Int J Radiat Oncol Biol Phys 2013; 87: 636-637.

18. Delishaj D, Laliscia C, Manfredi B et al. Non-melanoma skin cancer treated with high-dose-rate brachytherapy and Valencia applicator in elderly patients: a retrospective case series. J Contemp Brachytherapy 2015; 7: 437-444.

19. Barami KK. CyberKnife radiosurgery for management of intracranial perineural spread of cutaneous malignancies. J Clin Neurosci 2010; 17: 1322-1324.

20. Goyal U, Kim Y, Tiwari HA et al. A pilot study of ultrasoundguided electronic brachytherapy for skin cancer. J Contemp Brachytherapy 2015; 7: 374-380.

21. Ballester-Sánchez R, Pons-Llanas O, Candela-Juan C et al Electronic brachytherapy for superficial and nodular basal cell carcinoma: a report of two prospective pilot trials using different doses. J Contemp Brachytherapy 2016; 8: 48-55.

22. Bhatnagar A, Loper A. The initial experience of electronic brachytherapy for the treatment of non-melanoma skin cancer. Radiat Oncol 2010; 5: 87.

23. Ballester-Sánchez R, Pons-Llanas O, Candela-Juan C et al. Efficacy and safety of electronic brachytherapy for superficial and nodular basal cell carcinoma. J Contemp Brachytherapy 2015; 7: 231-238.

24. Pons-Llanas O, Ballester-Sánchez R, Celada-Álvarez FJ et al. Clinical implementation of a new electronic brachytherapy system for skin brachytherapy. J Contemp Brachytherapy 2015; 6: 417-423.

25. Arterbery VE, Watson AC. An electronic brachytherapy technique for treating squamous cell carcinoma in situ of the digit: a case report. BMC Res Notes 2013; 6: 147.

26. Treating with eBx. Xoft Inc. Available at: http:/ / www.xoftinc.com/treating_derma.html (accessed 24 October 2014).

27. Ballester-Sánchez R, Pons-Llanas O, Llavador-Ros M et al. Depth determination of skin cancers treated with superficial brachytherapy: ultrasound vs. histopathology. J Contemp Brachytherapy $2015 ; 6:$ 356-361.

28. Ma CM, Coffey CW, DeWerd LA et al. TG-61 AAPM protocol for 40-300 kV x-ray beam dosimetry in radiotherapy and radiobiology. Med Phys 2001; 28: 868-893.

29. Binder M, Puespoeck-Schwarz M, Steiner A et al. Epiluminescence microscopy of small pigmented skin lesions: short term formal training improves the diagnostic performance of dermatologists. I Am Acad Dermatol 1997; 36: 197-202.

30. Grin CM, Kopf AW, Welkovich B et al. Accuracy in the clinical diagnosis of malignant melanoma. Arch Dermatol 1990; 126: 763-766.

31. Mayer J. Systematic review of the diagnostic accuracy of dermatoscopy in detecting malignant melanoma. Med J Aust 1997; 167: 206-210.

32. Miller M, Ackerman AB. How accurate are dermatologists in the diagnosis of melanoma? Degree of accuracy and implications. Arch Dermatol 1992; 128: 559-560.

33. Wolf IH, Smolle J, Soyer HP et al. Sensitivity in the clinical diagnosis of malignant melanoma. Melanoma Res 1998; 8: 425-429.

34. Gambichler T, Regeniter P, Bechara FG et al. Characterization of benign and malignant melanocytic skin lesions using optical coherence tomography in vivo. J Am Acad Dermatol 2007; 57: 629-637.

35. O'Donnell AT, Kim CC. Update and clinical use of imaging technologies for pigmented lesions of the skin. Semin Cutan Med Surg 2012; 31: 38-44.

36. Rohrbach DJ, Muffoletto D, Huihui J et al. Preoperative mapping of nonmelanoma skin cancer using spatial frequency domain and ultrasound imaging. Acad Radiol 2014; 21: 263-270.

37. Wong S, Kaur A, Back M et al. An ultrasonographic evaluation of skin thickness in breast cancer patients after postmastectomy radiation therapy. Radiat Oncol 2011; 6: 9.

38. Kučinskiené $\mathrm{V}$, Samulènienè $\mathrm{D}$, Gineikienè $\mathrm{A}$ et al. Preoperative assessment of skin tumor thickness and structure using 14-MHz ultrasound. Medicina (Kaunas) 2014; 50: 150-155.

39. Bobadilla F, Wortsman X, Muñoz C et al. Pre-surgical high resolution ultrasound of facial basal cell carcinoma: correlation with histology. Cancer Imaging 2008; 8: 163-172.

40. Cuttino LW, Todor D, Rosu M et al. A comparison of skin and chest wall dose delivered with multicatheter, Contura multilumen balloon, and MammoSite breast brachytherapy. Int J Radiat Oncol Biol Phys 2011; 79: 34-38. 